\title{
COMPRENSIÓN Y PRODUCCIÓN DE ARGUMENTACIÓN ESCRITA EN ESTUDIANTES DE EDUCACIÓN PRIMARIA
}

\author{
COMPREHENSION AND PRODUCTION OF \\ WRITTEN ARGUMENTATION IN PRIMARY \\ STUDENTS
}

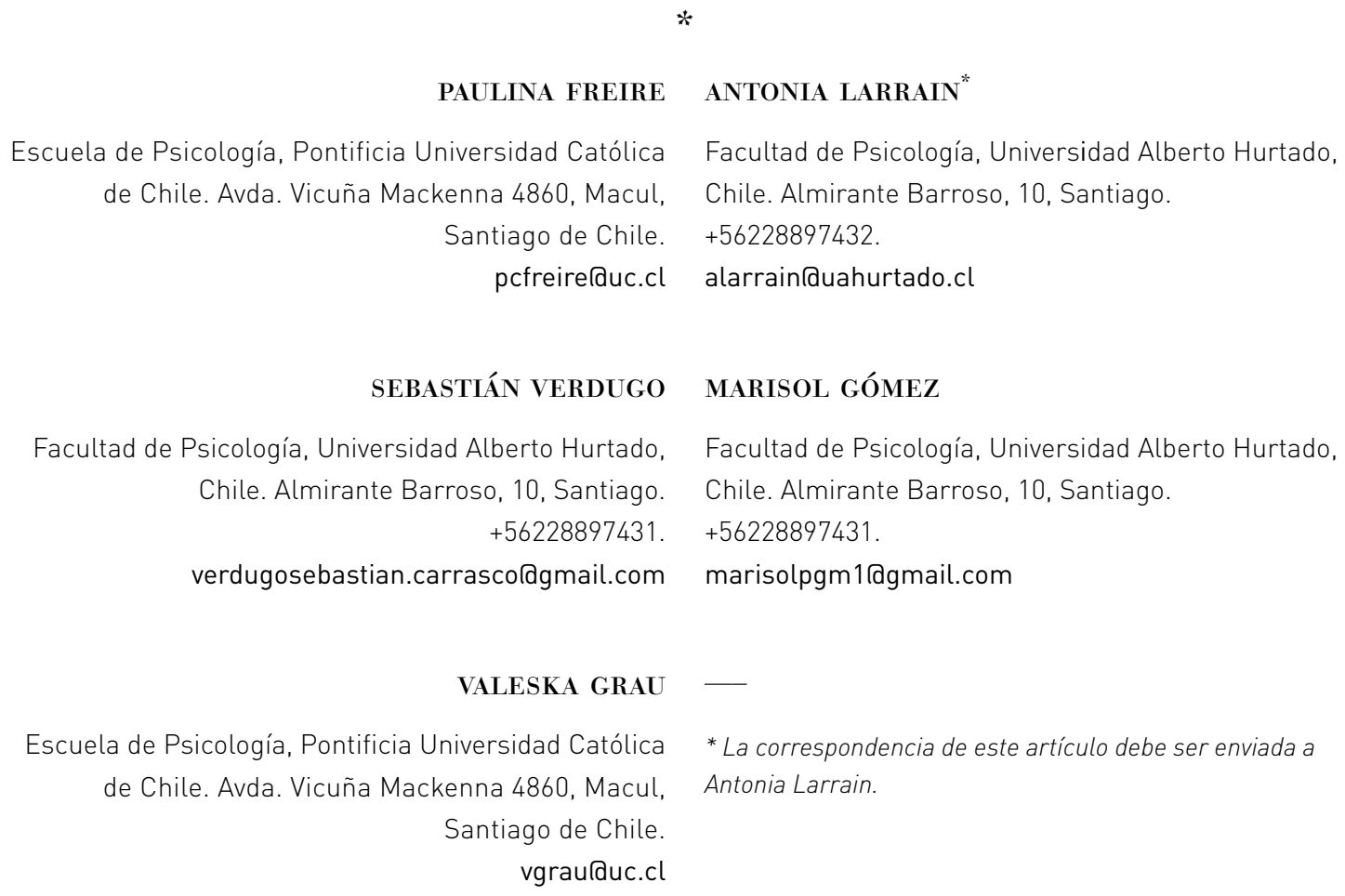

Este estudio fue posible gracias al apoyo financiero del Fondo de Investigación Científico y Tecnológico (FONDECYT) de Chile de ANID, proyecto 1170431. 
Ser capaz de comprender y producir argumentos, debe ser una de las competencias más importantes que un ciudadano digital tiene que desplegar actualmente. Sin embargo, poco sabemos respecto a las competencias y habilidades de comprender y producir argumentos de escolares en Chile. El objetivo del estudio es describir las habilidades de comprensión y producción de argumentos escrita de estudiantes chilenos de sexto año básico de educación subvencionada (privada y municipal), y su relación. Participaron 228 estudiantes (129 mujeres) de nueve establecimientos educacionales de Santiago de Chile. Los estudiantes respondieron dos tareas de comprensión y escritura de argumentos adaptadas de Reznitskaya y Wilkinson (2019). Los resultados muestran que si bien comprender argumentos en pro y contra a una posición es de equivalente dificultad, producir razones para sostener una perspectiva alternativa representa grandes dificultades para los y las estudiantes. Las habilidades de comprensión y producción correlacionan moderada pero significativamente, teniendo los estudiantes mayores dificultades al escribir que comprender argumentos. Además, existen diferencias de género a favor de las mujeres en escritura de argumentos. Se discuten las implicancias y significación de los resultados.

Palabras clave: comprensión, escritura, argumento, argumentación, contra-argumento.

To understand and formulate arguments, that is, to identify positions in a given a controversy, and to reconstruct the relationship between premises and conclusions; and to respond to them taking grounded point of views, is one of the most important competencies of digital citizen nowadays. However, there is scarce knowledge regarding adolescent argumentation skills (both in its comprehensive and productive aspects) in Chile. This study is aimed at describing Chilean sixth graders skills of argument comprehension and written production, and their relationship. 228 students (129 female) of nine schools in Santiago participated in the study. Students answered two tasks measuring argument comprehension and written production adapted from Reznitskaya and Wilkinson (2019). Results showed that while understanding pro and con arguments was equally challenging, writing reasons to support an alternative position was the most difficult goal to achieve. Regarding comprehension and writing relationships, there were moderate systematic and significant correlations between the achievement in the two tasks, but students had more difficulties with writing than understanding arguments. Moreover, girls outperformed boys in writing arguments. Results significance and implicancies are discussed.

Key words: comprehension, writing, argument, argumentation, counter-argument. 


\section{Introducción}

Ser capaz de comprender y producir argumentos, esto es, identificar posiciones en un debate, reconstruir la relación entre premisas y conclusión (Voss, Fincher-Kiefer, Wiley y Silfies, 1993), y responder tomando posición de manera razonada y razonable, debe ser una de las competencias más importantes que un ciudadano debe desplegar actualmente (Quintana y Correnti, 2019). En particular, esta competencia es interpelada constantemente en el caso de adolescentes y jóvenes, ante la demanda por el ejercicio de una ciudadanía digital, que supone involucramiento activo, crítico, responsable y respetuoso con tecnologías digitales en ámbitos de interés público (Frau-Meigs, O’Neill, Soriani y Tomé, 2017). Ser capaz de comprender lo que significa una toma de posición determinada, y de evaluar las razones o evidencia que la sostiene y, en función de eso, tomar una posición fundada, es hoy en día extremadamente valioso y tiene consecuencias nada despreciables para la vida en democracia (Gainer, 2012).

La pregunta es qué tan preparados están quienes hoy en día son adolescentes y jóvenes para participar sustentablemente de este mundo digital. La respuesta es que poco sabemos respecto a las competencias y habilidades de comprender y producir argumentos de adolescentes en Chile. Si bien la investigación internacional se ha concentrado en adolescentes (ver Rapanta, García-Mila y Gilabert, 2013), los estudios existentes en Chile se focalizan o bien en últimos años de escolaridad (Marinkovich, 2007) o educación universitaria (Larrain, Freire, Moretti, Requena y Sabat, 2015; Preiss, Castillo, Grigorenko y Manzi, 2013; Santibañez y Hample, 2015). Estos estudios han mostrado que las habilidades de argumentación se asocian al rendimiento en pruebas estandarizadas (Larrain et al., 2015; Preiss et al., 2013; Preiss, Castillo, Flotts y San Martín, 2013) lo que a su vez se relaciona con nivel socio-económico (Muñoz y Redondo, 2013). Esto hace sentido, pues las habilidades de argumentación tienen reducido espacio en el currículum nacional, y el sistema educativo entrega escasas oportunidades a estudiantes para el desarrollo de estas habilidades (Larrain, Freire y Howe, 2014; Preiss, Calcagni, Espinoza, Gómez, Grau, Guzmán, Müller, Ramírez y Volante, 2014). Así, es probable que el sistema educacional no pueda impactar la relación que estas habilidades podrían tener con las experiencias socio-culturales extra-escolares (Mercer, 2009).

Más allá de pruebas de pensamiento crítico, existen escasas alternativas disponibles para evaluar confiablemente las habilidades de argumentación de adolescentes al término de su educación primaria (Reznitskaya y Wilkinson, 2019), y la mayoría de los instrumentos que se han usado con fines de investigación se concentran en escritura argumentativa (ver Crowell y Kuhn, 2014; Iordanou, Kuhn, Matos, Shi y 
Hemberger, 2019; Knudson, 1992; Kuhn y Crowell, 2011; Kuhn, Shaw y Felton, 1997; Rapanta et al., 2013; entre muchos otros). La comprensión de argumentos ha sido mucho menos investigada, siendo una competencia clave para la participación actual de los y las jóvenes en el mundo social, económico y político.

Con el fin de contribuir en esta línea, este estudio reporta el uso de dos instrumentos para evaluar comprensión y producción de argumentos respectivamente en estudiantes de sexto básico de educación subvencionada en Chile, describiendo su desempeño en estas tareas y la relación entre estas sub-habilidades entre sí.

\section{Argumentación y habilidades de argumentación}

Las habilidades de argumentación pueden ser definidas como la capacidad de usar el lenguaje estratégicamente para formular argumentos y contra-argumentos, anticipar contra-argumentos y responderlos (Felton, 2004), y persuadir a interlocutores para hacerlos o bien cambiar de opinión o lograr un entendimiento (Santos y Leitao, 1999). Estas se despliegan en procesos de argumentación, es decir, prácticas sociales en donde hablantes intentan lidiar con asuntos controversiales (Coirier, Andriessen y Chanquoy, 1999), involucrándose en la producción e intercambio de argumentos para resolver o comprender sus diferencias (van Eemeren y Grootendorst, 1992). Para esto, los participantes se involucran en procesos de negociación (Leitão, 2000; Felton, 2004) marcados por usos de signos específicos: formulación puntos de vista y razones para sostenerlos, anticipación y respuesta a posiciones críticas y alternativas; y formulación de esas posiciones críticas (Leitão, 2000). Para Coirier et al. (1999), la argumentación se caracteriza por un continuo entre dos polos: la actividad de entregar evidencias para sostener una conclusión, y la acción dirigida a modificar creencias, conocimiento u otro, de una audiencia específica. No se trata de un tipo de actividad social, sino de un conjunto de actividades que justifican y producen la emergencia del uso de lenguaje argumentativo.

Siguiendo a autores como Billig (1996), Kuhn (2019) o Leitão (2000), concebimos la práctica de argumentación más allá de un proceso comunicativo, involucrando procesos de carácter intelectual y de construcción de conocimiento de naturaleza dialógica. De hecho, autores como Mercier y Sperber (2011) argumentan a favor de una visión argumentativa del razonamiento. Para Leitão (2000) la argumentación involucra y promueve procesos de construcción de conocimiento, a través de sus mecanismos semióticos: argumento, contra-argumento y respuesta. El argumento, entendido como una conclusión o punto de vista más apoyo o justificación, abre el discurso a la presencia de posiciones alternativas, aunque éstas no estén explícitamente formuladas. El contra-argumento, por su parte, representa una oposición 
explícita y fundamentada dirigida al argumento que promueve un giro atencional desde el objeto de discusión al fundamento de la propia postura, redirigiendo el pensamiento hacia sí mismo. Además, el contra-argumento, al ser la formulación de la alternativa opuesta, muestra claramente una dirección hacia la cual el pensamiento del hablante puede, eventualmente, cambiar. Por último, la respuesta, se pronuncia sobre ambas posiciones ya sea tomando partido por una, resolviendo, articulando, entre otros. Desde este punto de vista, y siguiendo a esta autora, entendemos la argumentación como un tipo de discurso con un potencial enorme de construcción de conocimiento en la medida que: 1) obliga al hablante a formular precisa y claramente sus ideas en base a evidencia; 2) entrega elementos discursivos que promueven la negociación de las ideas entre hablantes y la evaluación conjunta de éstas; 3 ) promueve el pliegue del pensamiento sobre sí mismo fomentando procesos meta-cognitivos de revisión y regulación de ideas.

Ahora bien, tanto la argumentación, como las habilidades de argumentación, pueden desplegarse de manera tanto oral como escrita, y aunque estos modos comparten características comunes (mecanismos semióticos) también suponen diferentes demandas cognitivas (Coirier et al., 1999). Mientras en la oralidad interlocutores co-construyen el proceso argumentativo, en la escritura el peso de la producción recae en un hablante quien debe anticipar la audiencia y su contexto (Larrain y Burrows, 2020). Además, la escritura involucra procesos de transformación del pensamiento (Bereiter y Scardamalia, 1987). Ahora bien, asumir una noción dialógica de la argumentación implica aceptar que esta es una práctica social que puede desarrollarse tanto entre dos o más interlocutores, como en el discurso de un mismo hablante, como en el caso de la escritura o del habla dirigida a uno mismo (ver Greco, 2017; Larrain, 2017). De esta manera, las habilidades de argumentación se juegan en ambos planos: tanto interpersonal, en la práctica de argumentación con otros hablantes; como intrapersonal, en la comprensión o producción argumentativa de un solo hablante.

Respecto a la noción de calidad argumentativa y sus criterios, reconocemos que la argumentación involucra diferentes dimensiones que la definen. Por una parte, está la dimensión estructural, es decir, la relación entre las distintas piezas de discurso que componen una cadena argumentativa. Al respecto, la contribución de Toulmin (1958) es clave pues ofrece distintas categorías para organizar y estandarizar un argumento. En particular, la relación entre evidencia y conclusión emerge como una clave de la argumentación de calidad, además de la cantidad de premisas, lo que se ha llamado complejidad del argumento (ver Stein y Bernas, 1999). 
Ahora bien, más allá de la presencia o ausencia de los distintos componentes estructurales, existe una dimensión lógico-pragmática relacionada con la relación entre evidencia y conclusión que se juega tanto en el contenido, es decir, en el valor de verdad de las premisas que componen un argumento; como en la relación lógica entre estas premisas y la conclusión que se pretende sostener. Le llamamos lógico-prágmática pues emergen de la lógica informal, y en general ponen en funcionamiento criterios de carácter paragmático. Las nociones de aceptabilidad, relevancia y suficiencia de las premisas son criterios claves (Govier, 2010). Desde este punto de vista, todo argumento debe juzgarse en base a qué tan aceptables son las premisas para determinada comunidad de hablantes; a si estas premisas sirven efectivamente desde el punto de vista de los hablantes para sostener la conclusión (o por en contrario, son irrelevantes o la debilitan); y en qué medida el conjunto de premisas son lógicamente suficientes para sostener una conclusión considerando un contexto de interlocución determinado (Coirier et al., 1999).

Dada la relación entre argumentación y construcción de conocimiento comentada más arriba, la dimensión epistémica también es relevante para definir un proceso de calidad. En el ámbito de la argumentación científica, por ejemplo, la calidad depende de la relación entre evidencia científica y conclusión, que se basa en aspectos lógicos-pragmáticos mencionados arriba, pero también depende de la calidad del conocimiento científico usado como evidencia (McNeill y Krajcik, 2008), lo que involucra una dimensión propiamente epistémica. Por otra parte, dado el rol que el aspecto dialéctico de la argumentación (contra-argumentación) juega en su potencial de construcción de conocimiento, la dimensión dialéctica ha sido considerado como parte central de buena argumentación (Santos y Leitão, 1999). Como vimos anteriormente, este criterio también responde a una dimensión epistémica pero no en el sentido de la calidad epistémica de las premisas, sino en el tipo de procesos psicológicos y cognitivos involucrados en las cadenas argumentativas.

Por último, aparece una dimensión estratégica: la capacidad de adecuar los recursos argumentativos al contexto retórico (Felton y Kuhn, 2001), entiéndase por esto, los propósitos de la argumentación (por ejempo, deliberar o persuadir), la característica de la audiencia y/o interlocutores, entre otros.

Si bien reconocemos las distintas dimensiones como relevantes para la calidad argumentativa, en este artículo enfatizaremos en el modelo de calidad las siguientes dimensiones: la dimensión estructural (identificación y producción de conclusiones y premisas y cantidad de estas); la dimensión lógico-pragmática, (aceptabilidad y relevancia); y la dimensión dialéctica (identificación y producción de contra-argumentos). 
Comprensión, producción y evaluación de argumentos

Las habilidades de argumentación además de involucrar distintas dimensiones (estructural, pragmática, lógica, epistémica, dialéctica y retórica), y modalidades comunicativas (oralidad y escritura), pueden entenderse como haciendo participar distintas sub-habilidades como comprensión, producción y evaluación de argumentos, y comprensión lectora y escritura. Se trata de sub-habilidades que emergen de manera interconectada e interdependiente, lo que dificulta no solo su conceptualización sino también su evaluación (Quintana y Correnti, 2019).

De acuerdo a Larson, Britt y Larson (2004) la comprensión de argumentos involucra una serie de procesos de lectura y razonamiento de alto orden, siendo el más básico la capacidad de representarse información esencial de la estructura de un argumento (conclusión y premisas). De esta manera, estos autores asumen que al comprender argumentos, las personas forman y usan esquemas argumentativos, es decir, generalizaciones de la estructura básica de un argumento, que les permite organizar la información. De acuerdo a Diakidoy, Ioannou y Cristodoulou (2017) el recuerdo preciso de los elementos de un argumento es clave para la comprensión (ver también Britt, Kurby, Dandotkar y Wolfe, 2008), lo que varía según el propósito que hablantes sostienen al comprender, a favor de un propósito evaluativo en comparación con uno de comprensión. Más aún, en general existe evidencia que muestra que el recuerdo preciso de un argumento es sorprendentemente difícil incluso para estudiantes universitarios (Britt et al., 2008). Ahora bien, y considerando la literatura en comprensión narrativa (Van den Broek, 1994), es posible pensar que comprender un argumento es más que identificar información básica, sino que implica reconstruir una representación coherente de una cadena argumentativa. Esto involucraría además de recuerdo, inferencias causales y evaluativas, que permiten identificar premisas implícitas y reconstruir su relación con las conclusiones de un texto, sea este oral o escrito. Esta reconstrucción además involucra la evaluación de la plausibilidad de las premisas y de la consistencia de estas entre entre sí, entre estas y la conclusión, y entre estas y creencias o conocimiento previo (Wolfe, Shawna y Taylore, 2013). Richter y Maier (2017) plantean que la evaluación de plausibilidad involucra tanto procesos pasivos, tácitos y automáticos de monitoreo epistémico, como procesos estratégicos, voluntarios y activos de elaboración de inconsistencias.

Por su parte, y siguiendo las dimensiones descritas en la sección anterior, la producción de argumentos involucraría la habilidad de comprender controversias y tomar posiciones en estas. Además, involucra formular estas posiciones con precisión y la capacidad de sostenerlas estratégicamente formulando premisas aceptables, relevantes y suficientes (dimensión estructural, lógico-pragmática y epistémica). Esto 
implica, que la habilidad de producción de argumentos supone una evaluación tácita de la relación entre premisas y conclusión. Por último, la habilidad de producción argumentativa involucra anticipar e imaginar posiciones alternativas y contra-argumentos, y responder efectivamente a estos (dimensión dialéctica), considerando el contexto retórico dado (dimensión estratégica).

Por último, la evaluación de argumentos involucra tomar una posición respecto a la aceptabilidad, relevancia y suficiencia de premisas en relación a determinada conclusión (Larson, Britt y Kurby, 2009), o la habilidad de juzgar si una conclusión se sigue de determinadas premisas (Quintana y Correnti, 2019). Esto implica representarse tanto la conclusión como el conjunto de premisas en juego; recordar y activar conocimiento relevante; articularlas con creencias y conocimiento; y analizarlas lógica y semánticamente en esa articulación.

En su conjunto, estas tres sub-habilidades aunque se pueden atribuir a individuos concretos, son esencialmente actividades sociales, interdependientes del contexto retórico en que se insertan, lo que incluye el tipo de hablantes y audiencia; objetivos de la interacción y de la tarea; características del asunto a discutir, entre otros. Por esta razón es ampliamente reconocida la dependencia que tiene el desempeño en tareas de argumentación del contexto retórico (Coirier et al., 1999; Leitão, 2003). Diversos autores han mostrado cómo las instrucciones explícitas y claras (Gilabert, García-Mila y Felton, 2013; Santos y Leitao, 1999), los objetivos (Garcia-Mila, Gilabert, Erduran y Felton, 2013), la familiaridad con la situación y audiencia (Stein y Miller, 1993), la debatibilidad del tema (Golder y Pouit, 1999), entre otros aspectos, modulan el despliegue de habilidades de argumentación impactando su posible evaluación.

Ahora bien, se piensa que la evaluación y comprensión de argumentos están íntimamente relacionados (Britt et al., 2008; Larson et al., 2009) e incluso podría se parte de un mismo constructo (Quintana y Correnti, 2019). Respecto a la relación entre comprensión y producción, la literatura también sugiere una estrecha relación involucrando tanto aspectos que se sobrelapan, como conocimiento de esquemas argumentativos, como aspectos en que se diferencian, como los procesos cognitivos y comunicativos que ponen en juego (Parodi, 2007). La evidencia disponible apoya esta idea, mostrando correlaciones altas y significativas entre ambos procesos, a la vez que sugieren cierta independencia. Parodi (2007) muestra correlaciones de 0,79 entre las habilidades de estudiantes chilenos de secundaria de inferir la tesis, argumento y conclusión de un texto, y formularlos en textos argumentativos. Reznitskaya y Wilkinson (2019) por su parte encuentran correlaciones moderadas entre comprender argumentos en un texto y producirlos, en estudiantes norteamericanos de 
primaria. Estas diferencias llaman la atención, dado que las tareas de Parodi (2007) y Reznitskaya y Wilkinson (2019) son similares. Esto puede deberse a la diferencia de temas utilizados y/o poblaciones evaluadas, o sugerir una menor diferenciación entre estas sub-habilidades a medida que los y las estudiantes avanzan en la experiencia educativa. Más aún, Parodi (2007) muestra que para estudiantes secundarios comprender argumentos sería más difícil que producirlos, y distintos estudios sugieren que el propósito y la enseñanza de escritura argumentiva orientaría y ayudaría la lectura de textos argumentativos (Newell, Beach, Smith y VanDerHeide, 2011).

Si el objetivo es fortalecer las habilidades argumentativas de estudiantes, avanzar en el conocimiento de la relación entre estas sub-habilidades es relevante (Newell et al., 2011; Parodi, 2007). Dado que la diferencia entre comprensión y evaluación no es clara, habiendo estudios que muestran unidimensionalidad (Quintana y Correnti, 2019), en este trabajo nos centraremos en la relación comprensión-producción.

\section{Habilidades de argumentación en adolescentes}

Si bien existe amplia literatura que reporta la dificultad de jóvenes e incluso adultos para argumentar, sobre todo de manera escrita (Felton y Kuhn, 2001; Knudson, 1992; Kuhn, 1991), existe evidencia consistente que muestra que incluso niños y niñas pequeños tienen conocimiento de argumentación y pueden producir todos los aspectos relevantes del proceso cuando el contexto es auténtico y tienen suficiente conocimiento de este (Stein y Bernas, 1999; Stein y Albro, 2001). Se reporta, sin embargo, una gran dificultad con la capacidad de adolescentes de representarse a interlocutores y audiencia (Stein y Albro, 2001), de formular críticas a los propios puntos de vista (Stein y Bernas, 1999), y de adecuarse estratégicamente al contexto retórico para persuadir (Felton y Kuhn, 2001). Ahora bien, estos estudios se han centrado en oralidad.

Las habilidades de argumentación escrita, si bien están íntimamente relacionadas con las habilidades de argumentación oral (Stein y Bernas, 1999), imponen dificultades específicas, pero también representan recursos claves de considerar en el mundo actual. Adolescentes y jóvenes hoy en día despliegan sus relaciones sociales más relevantes mediadas por un mundo virtual que se encarna a través de una compleja interrelación e interdependencia de medios escritos (chats, comentarios, textos), orales (videos) e imágenes (fotos) (Smith, Kiili y Kauppinen, 2016). Más aún, si bien toda argumentación se puede entender como un proceso dialógico (Billig, 1996; Leitão, 2000), siguiendo a Vygotski (1934/2001) la argumentación escrita se puede entender como un proceso cognitivamente complejo en la medida que demanda un 
proceso de doble abstracción en la que el hablante, junto con el procesamiento fonológico, debe representarse a su interlocutor, anticipando sus posibles respuestas de manera voluntaria y sistemática. Además, la escritura involucra procesos cognitivos sofisticadas de planificación, implementación y revisión (Coirier et al., 1999). Así, si bien uno podría pensar que las habilidades de argumentación orales podrían ser suficientes para que jóvenes se desplieguen en un mundo global, las especificidades de las habilidades escritas no deben quedar desatendidas.

En esta línea, desde el punto de vista de la relación entre premisas y conclusión, Knudson (1992) muestra que estudiantes de 12 años son capaces de construir argumentos escritos con mayor complejidad que estudiantes menores, pero tienen dificultades en integrarlos y organizarlos en un todo coherente para apoyar una conclusión. Desde el punto de vista de la estructura dialéctica, sabemos que cuando existen instrucciones apropiadas, hablantes desde los 12 y 13 años, pueden escribir ensayos integrando contra-argumentos a su propia perspectiva (Piolat, Roussey y Gombert, 1999), y que van avanzando a lo largo de la adolescencia en este sentido (Glassner y Schwarz, 2005). Aun así, existe asimetría en función de la cantidad de argumentos que se esgrimen a favor de la propia perspectiva en relación a la perspectiva alternativa (Stein y Bernas, 1999). En el contexto nacional, un estudio previo realizado en escuelas municipales de Santiago, mostró que estudiantes de quinto básico pueden construir argumentos simples, y anticipar elementos básicos de una perspectiva alternativa, sin embargo, solo un pequeño porcentaje logra elaborar contra-argumentos (Larrain, Freire y Lillo, 2013). Un estudio reciente, también en Chile, mostró que estudiantes de cuarto básico pueden, en su mayoría, formular puntos de vista y razones simples para sostenerlos, pero tienen gran dificultad de anticipar puntos de vista alternativos y contra-argumentos, elaborar argumentos estratégicamente para persuadir y evaluar el poder persuasivo de argumentos (Larrain y Singer, 2020). Más aún, el estudio mostró que el género y dependencia educativa predice el rendimiento en escritura argumentativa: mujeres y estudiantes de escuelas municipales tienen un desempeño sistemáticamente mejor que sus pares hombres o de escuelas subvencionadas de dependencia privada.

Ahora bien, lo que sabemos respecto a las habilidades de argumentación escrita de adolescentes se centra casi exclusivamente en conocimiento de argumentación y capacidad para desempeñarse en escritura argumentativa (Rapanta et al., 2013). Es muy escaso y difícil de rastrear el reporte de resultados ligado a las habilidades de comprensión de argumentos escritos (Larson, Britt y Larson, 2004). Stein y Bernas (1999) reportan resultados de evaluación de comprensión argumentativa y cómo en esta influye la posibilidad de recordar y conocer con precisión creencias y supuestos 
de personas que sostienen metas distintas a las propias, pero se centran sobre todo en contextos de interacción cara a cara. En uno de los escasos estudios en comprensión argumentativa en adolescentes, Chambliss y Murphy (2002), reportaron que estudiantes de cuarto y quinto grado logran representar un texto argumentativo como una estructura jerárquica global, aunque algunos solo lo hacen como un listado de ideas sin articulación; sugieren que la familiaridad con el contenido del texto influye positivamente en el desempeño. Parodi (2007) muestra cómo estudiantes secundarios en Chile muestran importantes dificultades para inferir la tesis, argumento y conclusión de un texto, dificultades que son aún mayores que la producción argumentativa. Por último, resultados en educación universitaria muestran que la comprensión argumentativa es una competencia que incluso a este nivel se encuentra subdesarrollada (von der Mühlen, Richter, Schmid y Berthold, 2019). Larson, Britt y Larson (2004), encontraron que estudiantes universitarios tenían dificultades para identificar elementos argumentativos claves; en particular, no identificaban todas las razones que sostienen una posición, seleccionaban premisas que no se relacionaban aceptablemente con una posición y, a menudo, confundían los contra-argumentos con posiciones centrales.

\section{Evaluación de habilidades de argumentación}

La producción argumentativa escrita se ha evaluado típicamente a través de ensayos argumentativos escritos, en donde se entregan instrucciones explícitas a las personas para tomar una posición y elaborar argumentos y contrargumentos, en función de un texto que varía en extensión y en el que se presenta un tema polémico (Kuhn, Shaw, y Felton, 1997; Kuhn, Goh, Iordanou y Shaenfield, 2008; Larrain et al., 2015; Parodi, 2007; Reznitskaya, Anderson, McNurlen, Nguyen-Jahiel, Archodidou y Kim, 2001). Además de estas tareas, y de manera menos frecuente, la escritura argumentativa se ha evaluado con tareas que involucran distintas situaciones controversiales con demandas puntuales respecto a tomar posición, y formular argumentos y contra-argumentos (Larrain y Singer, 2020); o tareas en las que se les solicita a participantes que formulen cadenas argumentativas a través de la representación de la continuación de un diálogo entre participantes respecto a un tema controversial (Kuhn, Zillmer, Crowell y Zavala, 2013).

La comprensión de argumentos, por su parte, se ha evaluado principalmente a través de la presentación de argumentos simples luego de lo cual se pide identificar posición y razones (Britt et al., 2008: Quintana y Correnti, 2019; Larson et al., 2004). También se ha evaluado a través de la presentación de distintos tipos de proposiciones luego de lo cual se solicita distinguir argumentos de descripciones o explicacio- 
nes (Quintana y Correnti, 2019). En menor medida, se han usado también textos argumentativos más extensos luego de lo cuales se les solicita a las personas responder preguntas centradas en la identificación de las distintas partes de los argumentos involucrados (Parodi, 2007). Diakidoy et al. (2017) y Chambliss y Murphy (2001) evaluaron comprensión argumentativa a través de la solicitud de reproducción de los argumentos involucrados en textos breves, justo después de leerlos.

Ahora bien, aún cuando se puede percibir cierta consistencia en las maneras de evaluar comprensión y producción de argumentación escrita, prácticamente no existen evaluaciones de habilidades argumentativas con medidas de confiabilidad y validez disponibles. Probablemente esto se deba a que estas habilidades son altamente sensibles al contexto retórico (Coirier et al., 1999; Leitão, 2003), lo que genera problemas en la interpretación de resultados y desafía la posibilidad de desarrollar mediciones estandarizadas que funcionen de manera estable en distintos grupos de personas. De hecho, existe un amplio consenso respecto a que evaluar las habilidades argumentativas es muy difícil, pues hay un riesgo alto de atribuir a una capacidad individual características de las tareas o contexto en que se pide argumentar (Leitão, 2003).

Una excepción a esto es el trabajo de Reznitskaya y Wilkinson (2019), quienes desarrollaron dos tareas de evaluación de habilidades argumentativas para estudiantes de primaria: una de comprensión y otra de producción argumentativa. La primera trata de textos breves que involucran situaciones controversiales, luego de lo cual se debe identificar la posición principal y alternativa, y las razones que las apoyan. La segunda, trata de una situación controversial que involucra un dilema moral ante lo cual se solicita tomar una posición y formular argumentos y contra-argumentos. El modelo de calidad involucrado combina diferentes dimensiones: valora la presencia y cantidad de razones identificadas y producidas (dimensión estructural); evalúa la relación de estas razones con las conclusiones que pretenden sostener (dimensión lógico-pragmática); y valora positivamente la identificación y formulación de posiciones alternativas y contra-argumentos (dimensión dialéctica). Cada tarea a través de aplicaciones con estudiantes de quinto año de educación primaria. Los autores reportan medidas aceptables de confiabilidad inter-jueces $(r>0,76)$ y de consistencia entre dos mediciones con muestras diferentes. Además, como medidas de validez muestran correlaciones moderadas pero significativas entre las tareas de producción y comprensión $(r=0,30-0,33, p$ $<0.01)$. Mujeres obtendrían puntajes significativamente más altos en ambas tareas solo en una de las muestras, y no se reportaron diferencias por grupo étnico (blancos y no blancos). 
Sabemos que las habilidades de argumentación son sensibles a la enseñanza (Felton, 2004; Glassner y Schwarz, 2005; Hefter, Berthold, Renkl, Riess, Schmidt y Fries, 2014; Kuhn y Crowell, 2011; Kuhn y Udell, 2003; Reznitskaya, et al. 2001; Schworm y Renkl, 2007; Weinberger, Stegmann, y Fischer, 2010), pero toda enseñanza requiere conocer su punto de partida. Considerando la relevancia para la vida en democracia en los próximos años, y la necesidad de llegar a acuerdos para enfrentar problemas y desafíos de magnitudes enormes, como el cambio climático, es central conocer las competencias no solo de producción, sino de comprensión de argumentos de estudiantes en edades clave para su desarrollo. Considerando que la evidencia no es conclusiva respecto a diferencias de género en estas habilidades, es relevante explorar su existencia. Por último, para diseñar acciones pedagógicas es relevante profundizar en las relaciones entre las sub-habilidades de comprensión y argumentación escrita.

Con el fin de contribuir en esta línea, este estudio reporta el uso de dos instrumentos adaptados de Reznitskaya y Wilkinson (2019) para evaluar comprensión y producción de argumentos respectivamente, y su relación, en estudiantes de sexto básico de educación subvencionada en Chile. El objetivo del estudio es describir las habilidades de comprensión y producción escrita de argumentos de estudiantes chilenos de educación subvencionada (privada y municipal) que cursaron sexto año básico entre 2017 y 2018, y explorar la relación entre estas sub-habilidades. Las preguntas que guiaron el estudio fueron: (1) ¿Cuál es el desempeño de estudiantes chilenos de educación subvencionada (privada y municipal) en sexto año básico en tareas de comprensión y producción de argumentos? (2) ¿Cómo se relacionan entre sí las sub-habilidades de comprender y producir argumentos? (3) ¿Existen diferencias significativas en el desempeño de estudiantes de acuerdo al género de los participantes?

\section{Método}

Para responder las preguntas de investigación realizó un estudio descriptivo correlacional.

\section{Participantes}

Se realizó un muestreo no probabilístico por conveniencia, invitando a participar a establecimientos educacionales subvencionados de Santiago dispuestos a participar en el estudio, algunos con los que ya se tenía contactos previos. Participaron 232 estudiantes (129 mujeres) de sexto año básico de 9 establecimientos subven- 
cionados (2 particulares) de la Región Metropolitana, Chile. Los establecimientos atendían una importante proporción de estudiantes vulnerables (entre 35,61\% y $84.2 \%$ ), y su rendimiento en la prueba que mide a nivel nacional conocimientos (SIMCE) en lenguaje el mismo nivel estaba 30 puntos el promedio nacional. La distribución de estudiantes según establecimiento se presenta en la tabla 1. Para invitarlos a participar, primero se contactó a directores de establecimientos quienes autorizaron el proceso de consentimiento y asentimiento informado, que se realizó con apoyo de los docentes a cargo de los respectivos cursos. Los estudiantes participantes, quienes entregaron su asentimiento y el consentimiento de sus padres, representan el 52,7\% del total de estudiantes matriculados en las respectivas clases.

\section{Tabla 1.}

Distribución de estudiantes según establecimientos educacionales, índice de vulnerabilidad y puntaje SIMCE de lenguaje 2018.

\begin{tabular}{|c|c|c|c|}
\hline Colegios & IVE & SIMCE & $\mathrm{n}$ \\
\hline 1 & 77,6 & 244 & 33 \\
\hline 2 & 93,4 & 221 & 20 \\
\hline 3 & 61,2 & 259 & 26 \\
\hline 4 & 79,9 & 229 & 17 \\
\hline 5 & 18 & 218 & 16 \\
\hline 6 & 81,5 & 244 & 13 \\
\hline 7 & 87,2 & 213 & 58 \\
\hline 8 & 78,7 & 252 & 31 \\
\hline 9 & 35,6 & 270 & \\
\hline
\end{tabular}

Nota. IVE = Índice de vulnerabilidad del establecimiento educacional 2018 según la Junta Nacional de Auxilio y Becas de Chile JUNAEB; SIMCE = Puntaje SIMCE de lenguaje de 6to básico del establecimiento año 2018.

\section{Procedimiento}

Los datos fueron recolectados en dos rondas entre la primavera del año $2017 \mathrm{y}$ el otoño del año 2018 como parte de un proyecto mayor que involucró medidas e intervención en aprendizaje en ciencias. Los y las estudiantes contestaron dos pruebas. En la primera ronda (105 estudiantes) lo hicieron en dos sesiones independientes (una para evaluar comprensión de argumentos y otra para evaluar producción de argumentos) luego de contestar una prueba de respuesta múltiple de 
ciencias. En la segunda ronda (127) realizaron las dos pruebas de argumentación en la misma sesión. Las pruebas fueron tomadas por el equipo de investigación en horario regular de clases y demoraban aproximadamente entre 20 y 30 minutos cada una. Posterior al análisis de desempeño, los establecimientos recibieron un informe de desempeño de los estudiantes anonimizado y agregado.

Las pruebas y rúbricas de corrección fueron adaptadas de las tareas desarrolladas por Reznitskaya y Wilkinson (2019), ya que, por un lado, se trata de uno los pocos instrumentos disponibles con medidas psicométricas, y por otro, reportan buenos índices de confiabilidad inter-jueces y validez concurrente. Además, estas tareas asumen un modelo de calidad basado en una concepción dialógica-dialéctica de la argumentación la que es coherente con el nuestro explicitado en la introducción. Luego de obtener permiso de la autora principal para el uso de las pruebas, estas fueron traducidas y piloteadas con 20 estudiantes del mismo nivel para indagar comprensibilidad e interés. Se adecuaron las pautas de corrección. Respecto a la rúbrica de la tarea de escritura de argumentos se separó un criterio que evaluaba claridad y estructura en el instrumento original, en dos. A partir de la pauta de corrección original de la tarea de comprensión de argumentos, se desarrolló una rúbrica con cuatro criterios. Las pruebas y rúbricas se validaron externamente por dos expertas en el área, quienes realizaron comentarios relevantes, especialmente focalizados en simplificación de los textos y rúbricas. La versión final de cada prueba se describe a continuación.

\section{Instrumentos}

Prueba de escritura de argumentos.

Como se mencionó, se adaptó la versión traducida de la tarea de escritura argumentativa de Reznitskaya y Wilkinson (2019). En términos generales, la tarea utilizada consistió en la lectura de un cuento breve (623 palabras) llamado "Una carrera de autitos de madera" en la que el personaje de un niño es enfrentado a un dilema moral y tiene que tomar una decisión entre dos posiciones (delatar o no a un compañero que hizo trampa). La lectura la realizaron co-autores a toda la clase. Luego de leer, se solicitó a cada estudiante que individualmente escribiera un texto planteando su opinión respecto a lo que ellos harían en el lugar del personaje enfrentado al dilema moral. Las instrucciones específicas se incluyen en la tabla 2. 
84 - Cogency, Journal of reasoning and argumentation

\section{Tabla 2.}

Instrucciones tarea de escritura de argumentos

\section{¿Debe Juan alertar al profesor acerca de lo que hizo Tomás?}

Por favor escribe lo que piensas para explicar por qué TÚ crees que Juan debiera hacerlo o por qué no debiera hacerlo.

- Recuerda expresar claramente tu opinión, presentando razones y evidencias.

- Recuerda también pensar por qué otras personas podrían estar en desacuerdo contigo y cómo podrías responderles.

- No olvides escribir una conclusión.

Esfuérzate al máximo y escribe lo más que puedas. Puedes releer la historia si deseas.

Los ensayos fueron corregidos en base a una rúbrica de corrección con cuatro niveles (no logrado, parcialmente logrado, logrado y sobresaliente) expresados en números entre $0 \mathrm{y}$ 3 , a excepción de los criterios de estructura y claridad que tienen tres niveles entre 0 y 2. Los criterios de evaluación se describen en su puntaje máximo en la tabla $3^{1}$.

\section{Tabla 3.}

Criterios rúbrica de corrección tarea de escritura de argumentos, puntaje máximo

1. Justificación de la postura elegida: Justifica la posición inicial entregando más de una razón de calidad en la medida que son aceptables y relevantes.

2. Perspectiva alternativa: Desarrolla la perspectiva alternativa o contraria entregando más de una razón de calidad en la medida que son aceptables y relevantes.

3. Conclusión: Sostiene o defiende una posición y explica por qué la postura elegida es más razonable, o las perspectivas alternativas no son aceptables. Adicionalmente, compara las posiciones desarrolladas y elige una de ambas posturas en base a su exposición.

4. Claridad del lenguaje: El ensayo, en general, posee un lenguaje claro y aceptable, para el desarrollo de las ideas.

5. Claridad de la estructura: El ensayo presenta, en general, una estructura coherente, en la medida que marca las tres partes del ensayo en su forma canónica.

1 La rúbrica completa de corrección está disponible para ser solicitada a la autora correspondiente. 
Dos psicólogos educacionales fueron entrenados por la autora correspondiente del artículo, y codificaron independientemente el 30\%, respectivamente en rondas sucesivas. Los índices de Kappa Cohen de las últimas rondas todos buenos $(p<, 01)$ : dos criterios $=\mathrm{K}>, 63$; un criterio $=\mathrm{K}=, 75$; dos $=\mathrm{K}>, 89$. Todos los desacuerdos fueron discutidos y acordados. El resto de las pruebas fueron corregidas en proporciones similares por cada corrector.

Prueba de comprensión de argumentos

Se adaptó la versión traducida de la tarea de comprensión argumentativa de Reznitskaya y Wilkinson (2019). El estímulo para la tarea es un pasaje corto (507 palabras) llamado “¿Son los zoológicos buenos lugares para los animales?” en el cual dos niñas plantean dos posiciones opuestas respecto a si los zoológicos son buenos lugares para los animales y dan razones para apoyar sus posturas. Luego de que los estudiantes leen individualmente el texto, se les solicitó identificar y escribir una de las opiniones expresadas en el texto y enumerar todas las razones que justifiquen esa opinión; finalmente, debían reconocer la opinión alternativa expresada en el texto y presentar todas sus razones (ver tabla 4).

Tabla 4.

Instrucciones tarea de comprensión de argumentos

¿Son los zoológicos buenos lugares para los animales?

Por favor lee las instrucciones atentamente.

En el texto se presentan dos opiniones.

Opinión $\mathrm{N}^{\circ} 1$

En una oración y en tus propias palabras, por favor presenta una de las opiniones expresadas en el texto:

En tus propias palabras, por favor enumera todas las razones que justifican esta opinión:

1.

2.

3. 


\section{Opinión $\mathrm{N}^{\circ} 2$}

En una oración y en tus propias palabras, por favor presenta la otra opinión expresada en el texto:

En tus propias palabras, por favor enumera todas las razones que justifican esta segunda opinión:

1.

2.

3.

Las respuestas fueron corregidas en base a una rúbrica de corrección con cuatro niveles (no logrado, parcialmente logrado, logrado y sobresaliente) expresados en números entre 0 y 3 . Los criterios de evaluación se describen en su puntaje máximo en la tabla $5^{2}$.

\section{Tabla 5.}

Criterios rúbrica de corrección tarea de comprensión de argumentos, puntaje máximo

1. Primera posición: Reconoce y formula claramente una de las dos posturas (A o B) que se expresan en el texto.

2. Razones de primera posición: Identifica al menos cuatro razones señaladas explícitamente en el texto

3. Posición contraria: Reconoce y formula claramente la opinión contraria (según lo respondido en la pregunta 1) expresada en el texto.

4. Razones posición contraria: identifica al menos cuatro razones señaladas en el texto.

Dos psicólogos educacionales fueron entrenados por la autora principal del artículo, y codificaron independientemente el 29\%, respectivamente en rondas sucesivas. Los índices de Kappa Cohen de las últimas rondas todos excelentes $(\mathrm{K}>853, p<, 01)$. El resto de las pruebas fueron corregidas en proporciones similares por cada corrector.

\footnotetext{
2 La rúbrica completa de corrección está disponible para ser solicitada a la autora correspondiente.
} 
Análisis

Con el propósito de describir las habilidades de escritura y comprensión argumentativa de los y las estudiantes, en primer lugar, se analizaron descriptivamente los puntajes obtenidos en cada uno de los criterios evaluados. Luego, con el objetivo de explorar las relaciones entre los distintos aspectos evaluados, especialmente entre los criterios de las distintas tareas (comprensiva y productiva), se hizo un análisis de correlaciones bivariadas de Pearson entre los nueve aspectos. Posteriormente, para agregar los criterios de manera sumativa en puntajes totales, se realizó un análisis de componentes principales (ACP) con rotación oblicua (oblimin), ya que se esperaba que estos correlacionaran. Luego, se analizó la consistencia interna de cada uno de los componentes mediante alpha de Cronbach, y se presentaron los resultados descriptivos para los puntajes totales y las correlaciones bivariadas entre ellos. Por último, para conocer si las diferencias observadas en los puntajes promedio de escritura y comprensión argumentativa entre hombres y mujeres eran significativas, se estimaron modelos de regresión múltiple, con género como la variable independiente de interés. La regresión se condujo con el método jerárquico, siguiendo un procedimiento sugerido por Huang (2016) para controlar la varianza relacionada con los colegios. En un primer paso, se ingresó como predictor el colegio de procedencia, y en un segundo paso, se incorporó el género; ambas variables categóricas fueron codificadas como variables dummy, con el puntaje de los hombres como categoría de referencia para hacer la comparación. Los análisis fueron realizados utilizando la librería Psych (Revelle, 2019) del software estadístico R.

\section{Resultados}

¿Cuál es el desempeño de estudiantes chilenos de educación subvencionada (privada y municipal) en sexto año básico en tareas de comprensión y producción de argumentos?

En la prueba de escritura de argumentos, los y las estudiantes $(n=228)$ elaboraron textos breves de 89 palabras en promedio (con un rango entre 16 y 254 palabras). Respecto a los aspectos de la tarea evaluados, y como se observa en la tabla 6, el mayor puntaje promedio se obtuvo en la claridad del lenguaje (rango $=0-2$, rango teórico $=0-2$ ), criterio que presentó una distribución de los resultados fuertemente asimétrica hacia el extremo superior, ya que un 86,8\% de estudiantes logró escribir el ensayo con un lenguaje claro y aceptable para el desarrollo de las ideas (ver un ejemplo en figura 1). 
Tabla 6.

Estadística descriptiva, prueba de escritura de argumentos

\begin{tabular}{|c|c|c|c|c|c|c|c|c|c|c|c|}
\hline \multirow{2}{*}{ Colegio } & \multirow{2}{*}{$n$} & \multicolumn{2}{|c|}{ Justificación } & \multicolumn{2}{|c|}{ P. Alternativa } & \multicolumn{2}{|c|}{ Conclusión } & \multicolumn{2}{|c|}{ Claridad } & \multicolumn{2}{|c|}{ Estructura } \\
\hline & & M & DE & M & $\mathrm{DE}$ & M & DE & M & $\mathrm{DE}$ & M & DE \\
\hline 1 & 33 & 1,67 & 0,82 & 0,46 & 0,75 & 1,06 & 0,79 & 1,76 & 0,50 & 1,06 & 0,71 \\
\hline 2 & 20 & 1,50 & 0,76 & 0,85 & 0,75 & 0,25 & 0,55 & 1,95 & 0,22 & 0,85 & 0,59 \\
\hline 3 & 17 & 1,71 & 0,85 & 0,06 & 0,24 & 0,12 & 0,49 & 2,00 & 0,00 & 0,12 & 0,33 \\
\hline 4 & 26 & 1,54 & 0,51 & 0,15 & 0,37 & 0,73 & 0,83 & 1,92 & 0,39 & 0,65 & 0,75 \\
\hline 5 & 18 & 1,56 & 0,51 & 0,11 & 0,32 & 0,33 & 0,69 & 1,78 & 0,65 & 0,33 & 0,59 \\
\hline 6 & 16 & 1,88 & 0,50 & 0,19 & 0,54 & 0,81 & 0,83 & 2,00 & 0,00 & 0,69 & 0,60 \\
\hline 7 & 11 & 1,55 & 0,82 & 0,00 & 0,00 & 0,36 & 0,81 & 2,00 & 0,00 & 0,18 & 0,40 \\
\hline 8 & 56 & 1,59 & 0,63 & 0,57 & 0,85 & 0,91 & 0,64 & 1,64 & 0,59 & 1,00 & 0,54 \\
\hline 9 & 31 & 1,81 & 0,60 & 0,32 & 0,54 & 1,26 & 0,77 & 1,90 & 0,40 & 1,19 & 0,65 \\
\hline Total & 228 & 1,64 & 0,67 & 0,37 & 0,67 & 0,76 & 0,79 & 1,83 & 0,46 & 0,79 & 0,28 \\
\hline
\end{tabular}

En relación a la justificación de puntos de vista, la mayoría de estudiantes logró cumplir, al menos parcialmente, este criterio. Como se observa en la figura 2, un $38,6 \%$ justificó la posición inicial entregando como mínimo una razón, pero de baja calidad; un 50,9\% entregó al menos una razón de calidad aceptable y relevante, y un 7,9\% lo hizo entregando dos o más razones de este tipo. En contraste, el desarrollo de la postura alternativa obtuvo el promedio de logro más bajo de la prueba, con la mayoría de los puntajes distribuidos asimétricamente en el extremo inferior. Un $72,8 \%$ de estudiantes no logró argumentar considerando una perspectiva alternativa; el 18,4\% logró anticipar una o más razones para sostener una perspectiva alternativa, pero éstas fueron de baja calidad; solo un 7,9\% de estudiantes anticipó una perspectiva alternativa sosteniéndola con una razón aceptable y relevante; y menos de un $1 \%$ de estudiantes lo hizo entregando más de una razón de este tipo. También el puntaje promedio obtenido en la conclusión fue bajo. Un $44,3 \%$ de estudiantes no sostuvo claramente una posición a defender al modo de conclusión, o no se pudo suponer su alineación con ninguna de las posturas esgrimidas; un 36,4\% sostuvo o defendió una posición, pero no explicó por qué la postura elegida era más razonable o por qué las perspectivas alternativas no eran aceptables; solo un 18\%, logró explicar por qué finalmente la postura elegida era la más razonable; y tres personas $(1,3 \%)$ alcanzaron el nivel de logro más alto, al comparar las posiciones desarrolladas y elegir una de ambas posturas en base a su exposición. Por último, con respecto a la estructura del ensayo, un 49,6\% de estudiantes planteó una estructura coherente de ensayo, en la medida que marcaba dos de las tres partes del ensayo en su forma canónica, y un 14,9\% presentó las tres partes del ensayo (argumentación, contra-argumentación y conclusión). 


\section{Figura 1.}

Ejemplo de respuesta de tarea de escritura de argumentos

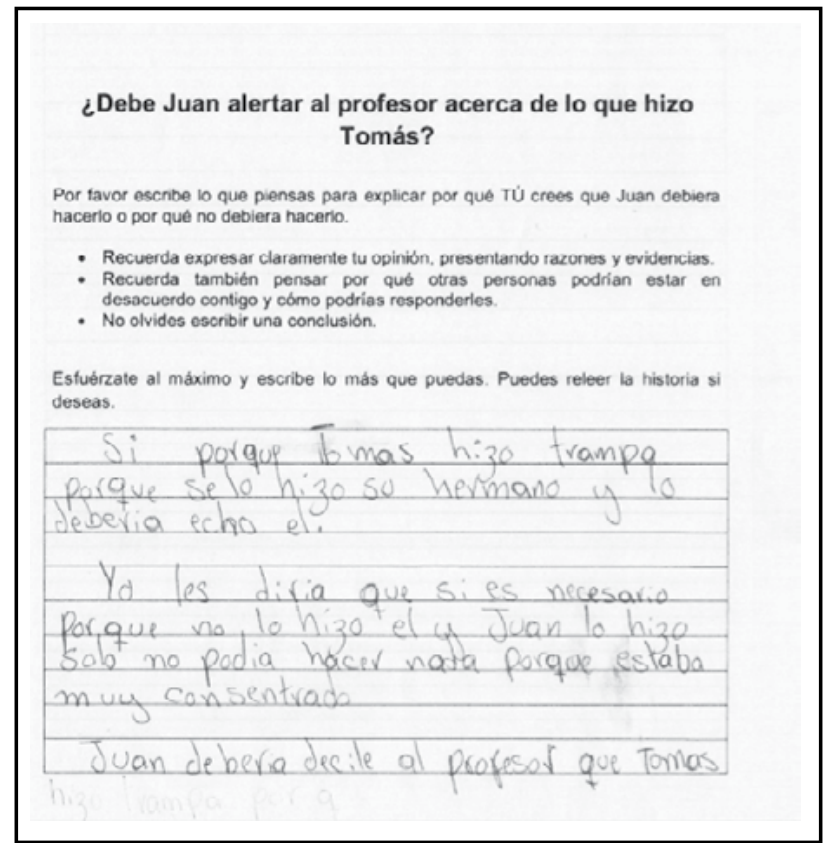

Figura 2.

Desempeño promedio estudiantes tarea de escritura de argumentos según criterios

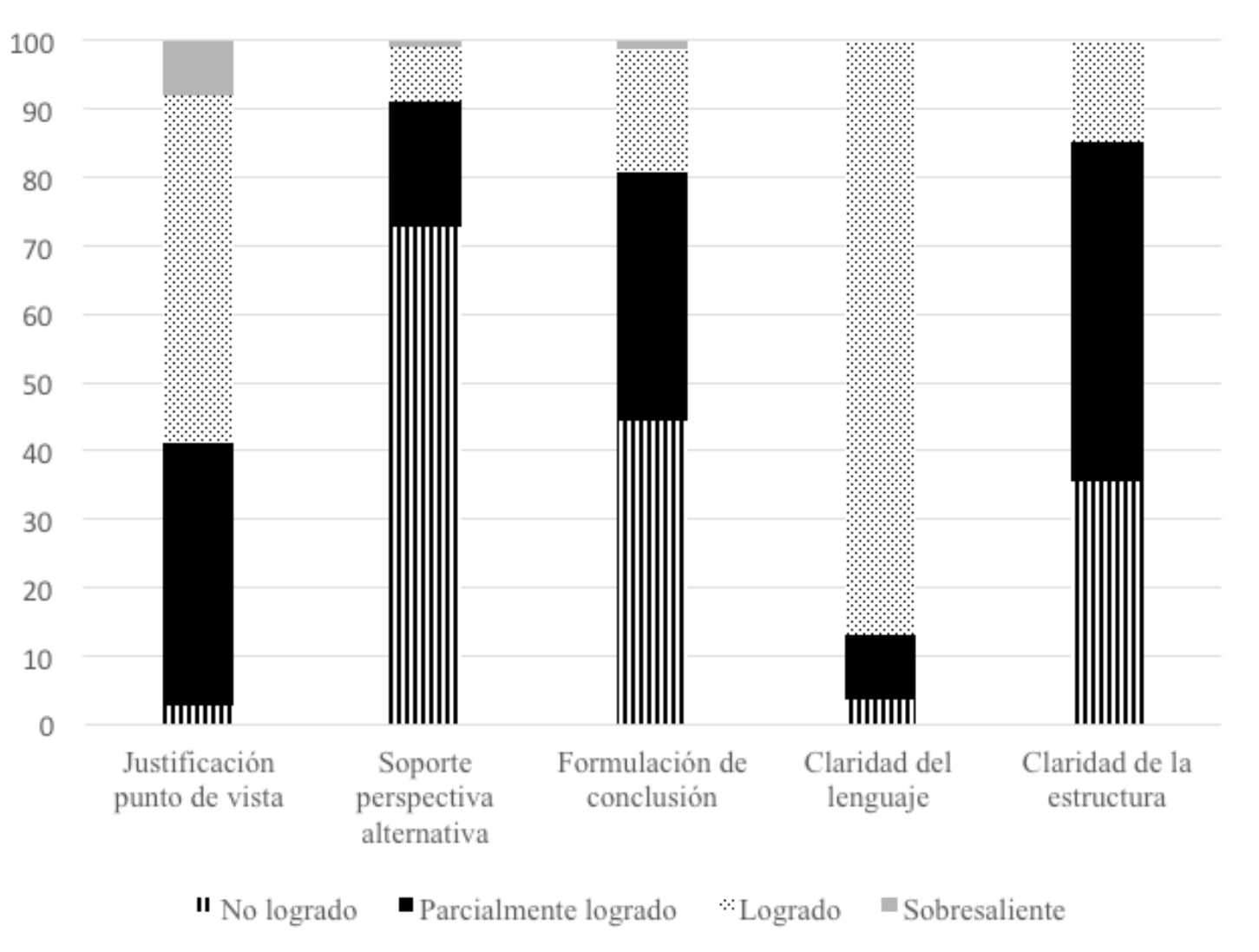


En la prueba de comprensión de argumentos, los y las estudiantes $(n=215)$ escribieron textos de 90 palabras, con un rango entre 7 y 161 palabras (ver ejemplo en figura 3). En promedio, se observan puntajes relativamente similares en los cuatro criterios de la prueba (ver tabla 7). Respecto al primer aspecto evaluado, un $56,7 \%$ de estudiantes logró identificar y presentar con sus palabras una de las posiciones del texto; y de ellas, el 30,2\% lo hizo claramente. Sin embargo, un 43,26\% no identificó explícitamente una de las dos posturas, y presentó en cambio razones para apoyar una posición implícita $(29,77 \%)$, o, en el nivel más bajo, una respuesta que no se relacionaba con una de las dos posturas del texto (por ejemplo, al plantear una posición distinta a las del texto, ver razón 3 fig. 3). Luego, frente a la solicitud de enumerar todas las razones del texto para justificar la posición identificada, la mayoría identificó alguna razón. Un 32,56\% presentó solo una razón, un 43,72\% reconoció entre dos a tres razones del texto, y sólo un 8,37\% alcanzó el nivel de logro más alto, enumerando cuatro o más razones. En relación con el tercer criterio que evalúa la identificación de la postura alternativa, el puntaje promedio observado fue ligeramente más bajo que al reconocer una primera posición. Un 55,8\% de estudiantes no logró dar cuenta de la postura alternativa presentada en el texto, mientras el 44,2\% restante sí la identificó; entre estos últimos, el 31,16\% lo hizo claramente. Por último, en el cuarto criterio se obtuvo un puntaje promedio levemente menor al resto de los aspectos evaluados. Mientras un 29,75\% de estudiantes no logró identificar razones, un 20,93\% identificó solo una de ellas, un 42,8\% reconoció entre dos a tres, y el 6,5\% alcanzó el mayor nivel de logro, dando cuenta de cuatro o más razones.

\section{Tabla 7.}

Estadística descriptiva, prueba de comprensión de argumentos

\begin{tabular}{|c|c|c|c|c|c|c|c|c|c|c|}
\hline \multirow{2}{*}{ Colegio } & \multirow{3}{*}{$\mathrm{n}$} & \multicolumn{3}{|l|}{ Posición A } & \multicolumn{2}{|l|}{ Razones A } & \multicolumn{2}{l|}{ Posición B } & \multicolumn{2}{l|}{ Razones B } \\
\cline { 3 - 11 } & & $\mathrm{M}$ & $\mathrm{DE}$ & $\mathrm{M}$ & $\mathrm{DE}$ & $\mathrm{M}$ & $\mathrm{DE}$ & $\mathrm{M}$ & $\mathrm{DE}$ \\
\hline 1 & 32 & 1,69 & 1,09 & 1,38 & 0,91 & 1,16 & 1,27 & 1,29 & 0,95 \\
\hline 2 & 20 & 2,10 & 1,12 & 1,60 & 0,82 & 1,95 & 1,19 & 1,75 & 0,79 \\
\hline 3 & 17 & 1,53 & 1,18 & 1,12 & 0,78 & 0,41 & 0,87 & 0,35 & 0,79 \\
\hline 4 & 26 & 2,27 & 0,87 & 1,65 & 0,75 & 1,88 & 1,24 & 1,50 & 0,91 \\
\hline 5 & 17 & 2,00 & 1,32 & 1,00 & 0,87 & 1,41 & 1,33 & 1,06 & 0,90 \\
\hline 6 & 16 & 2,19 & 1,11 & 2,06 & 0,93 & 2,06 & 1,12 & 1,56 & 0,89 \\
\hline 7 & 12 & 1,75 & 1,22 & 1,25 & 0,97 & 1,92 & 1,38 & 1,25 & 1,06 \\
\hline 8 & 46 & 1,20 & 0,65 & 1,30 & 0,70 & 1,09 & 0,96 & 1,00 & 0,89 \\
\hline 9 & 29 & 1,62 & 0,78 & 1,69 & 0,85 & 1,62 & 0,94 & 1,72 & 0,88 \\
\hline Total & 215 & 1,73 & 1,04 & 1,45 & 0,85 & 1,48 & 1,20 & 1,26 & 0,96 \\
\hline
\end{tabular}




\section{Figura 3.}

Ejemplo de respuesta de tarea de comprensión de argumentos

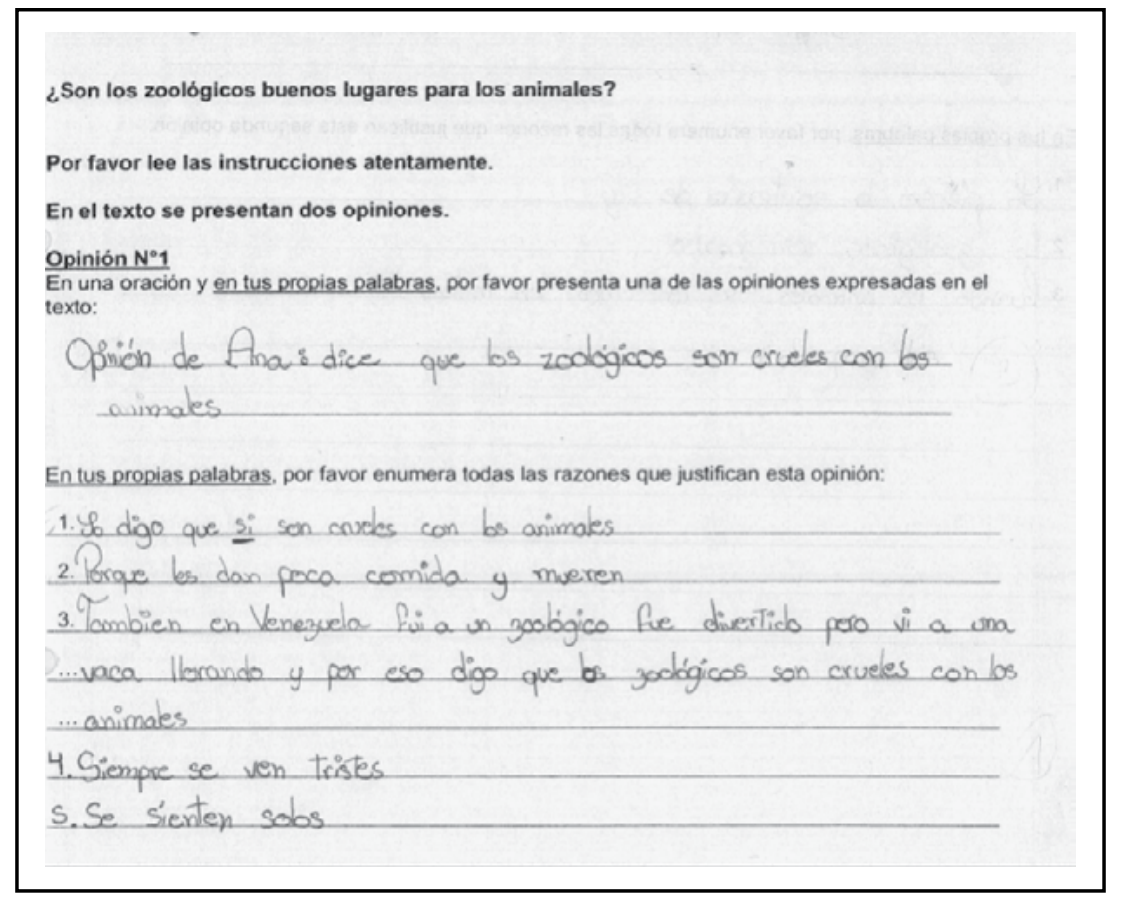

\section{Figura 4.}

Desempeño promedio estudiantes tarea de comprensión de argumentos según criterios

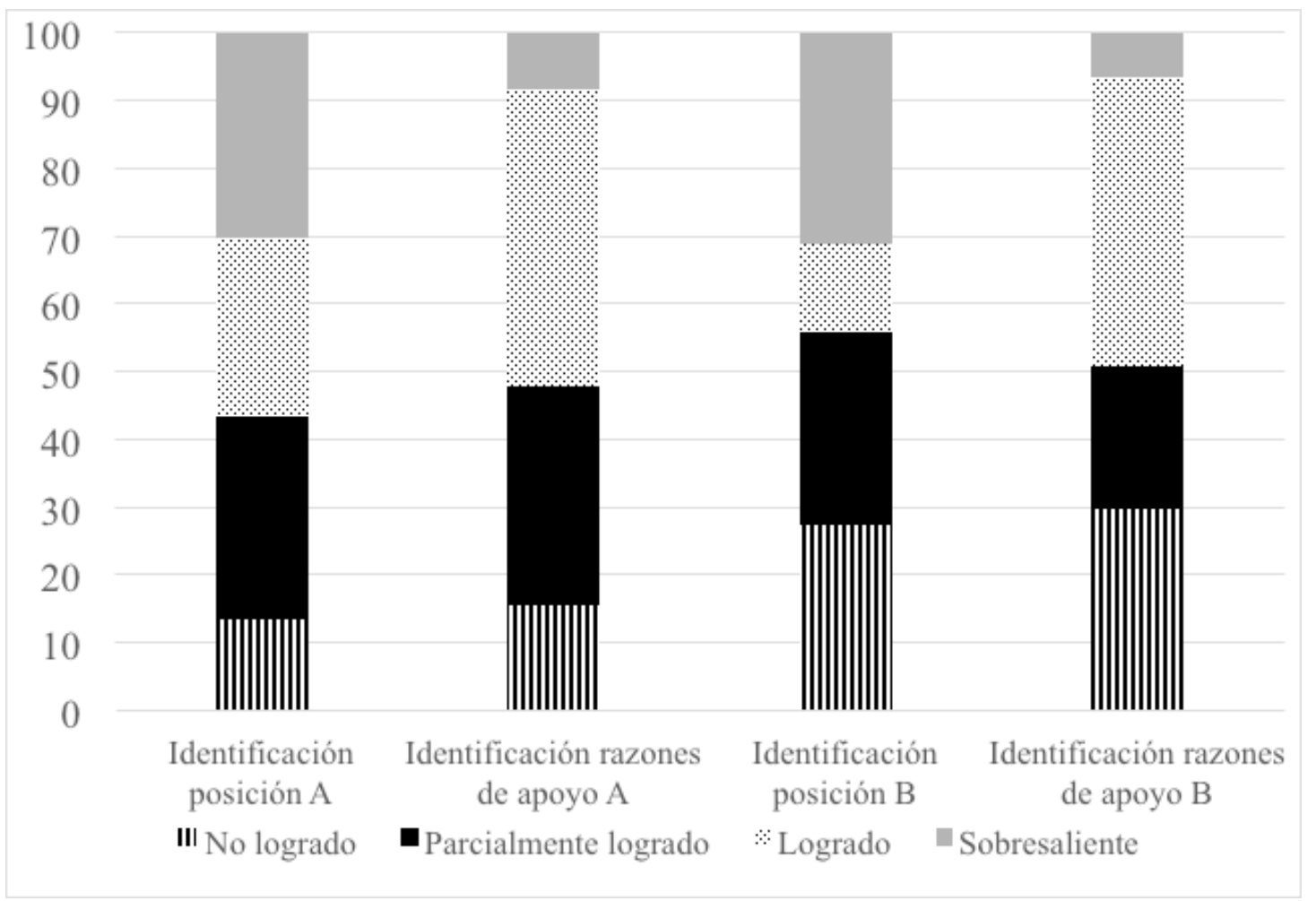


¿Cómo se relacionan entre sí las sub-habilidades de comprender y producir argumentos?

Para explorar las relaciones entre los distintos aspectos evaluados se analizaron sus correlaciones. Como es posible observar en la tabla 8 , hay correlaciones positivas entre criterios de la tarea de escritura. El desarrollo de una perspectiva alternativa correlaciona alta y significativamente con la claridad de la estructura del ensayo, lo mismo ocurre entre conclusión y estructura, mientras que perspectiva alternativa y conclusión correlacionan baja pero significativamente. Sin embargo, llama la atención que la justificación de razones de apoyo a un punto de vista no correlaciona significativamente con el desarrollo de una perspectiva alternativa, aunque la primera tiene una correlación baja con conclusión. También es posible observar que los puntajes en claridad del lenguaje no correlacionan con ningún otro criterio de escritura, con excepción de una correlación débil y positiva con la justificación de un punto de vista. Esta falta de relación podría deberse a los problemas con este criterio, ya que la mayoría de sus puntajes se distribuyó de manera muy asimétrica en el nivel de logro más alto.

Respecto a la tarea de comprensión, se observa que entre todos los criterios de esta prueba hay correlaciones positivas significativas de moderadas a altas, lo que sugiere que están evaluando aspectos de una misma dimensión. Al observar la relación entre ambas pruebas se observan correlaciones positivas significativas, aunque bajas entre algunos criterios. Es interesante notar que la justificación de razones de apoyo a un punto de vista (tarea de escritura) correlaciona directa y significativamente con la identificación de razones de apoyo tanto a un primer punto de vista como al punto de vista alternativo (tarea de comprensión); asimismo, la formulación de una conclusión correlaciona débil pero significativamente con tres de los cuatro criterios de la prueba de comprensión. Sin embargo, anticipar y desarrollar una perspectiva alternativa en la tarea de escritura no correlaciona con la comprensión de posiciones y razones, sean o no contrarias. 
Tabla 8.

Correlaciones bivariadas de criterios de tareas de escritura y comprensión de argumentos

\begin{tabular}{|c|c|c|c|c|c|c|c|c|}
\hline & 2 & 3 & 4 & 5 & 6 & 7 & 8 & 9 \\
\hline 1. Justificación & -.01 & $.17 * *$ & $.18^{* *}$ & .12 & .03 & $.31 * * *$ & 0,12 & $.21 * *$ \\
\hline $\begin{array}{l}\text { 2. Perspectiva alter- } \\
\text { nativa }\end{array}$ & & .19** & .01 & $.56 * * *$ &,- 01 & .12 & .05 & ,08 \\
\hline 3. Conclusión & & & , 00 & $.73^{* * *}$ &,- 07 &, $21^{* *}$ & , $21^{* *}$ & $.21^{* *}$ \\
\hline 4. Claridad & & & & .00 & .13 & .04 & $.17 *$ & $.18^{* *}$ \\
\hline 5. Estructura & & & & &,- 09 & $.22^{* *}$ & ,20** & $.22^{* *}$ \\
\hline 6. Posición A & & & & & & $.32^{* * *}$ &, $51 * * *$ &, $32^{* * *}$ \\
\hline 7. Razones PA & & & & & & & $.43^{* * *}$ &, $54^{* * *}$ \\
\hline 8. Posición B & & & & & & & &, $60^{* * *}$ \\
\hline 9. Razones PB & & & & & & & & \\
\hline
\end{tabular}

Nota. Los criterios 1-5 corresponden a la tarea de escritura: 1. Justificación de la postura elegida; 2. Desarrollo de una perspectiva alternativa; 3. Conclusión; 4. Claridad del lenguaje; 5. Claridad de la estructura argumentativa. Los criterios 6-9 corresponden a la tarea de comprensión argumentativa: 6. Posición A: Reconoce y formula una de las dos posturas del texo; 7. Razones PA: Identifica razones de primera posición; 8. Posición B: reconoce posición contraria; 9, Razones PB: Identifica razones de posición contraria. ${ }^{*} p<, 05 .{ }^{* *} p<, 01{ }^{* * *} p<, 001$

Para resumir las puntuaciones de los criterios en puntajes totales, se realizó un análisis de componentes principales (PCA) con rotación oblimin. Se incluyeron todos los criterios de ambas pruebas, con excepción de claridad del lenguaje, debido a su pronunciada asimetría y baja correlación con los otros criterios; además, este no evaluaba directamente un componente argumentativo. La prueba de esfericidad de Bartlett $\left(\chi^{2}(28)=556, \mathrm{p}<, 001\right)$ indicó que las correlaciones entre los elementos eran suficientes para el PCA. Un primer análisis para conocer los autovalores de cada componente mostró que tres de ellos cumplían con la regla de Kaiser de ser mayores a 1 para ser seleccionados; en cambio, el gráfico de sedimentación y el análisis paralelo sugerían la extracción de dos componentes. Debido a que estos últimos criterios son más recomendados por su precisión (Field, Miles y Field, 2012), se probó una solución rotada de dos componentes; además, esta decisión se apoyaba en la interpretabilidad de los resultados, ya que se esperaba encontrar una dimensión referente a la tarea de comprensión, y otra, a la producción argumentativa. Como se observa en la tabla 9, en el primer componente se agruparon todos los criterios de comprensión de argumentos, y en el segundo se agruparon tres criterios de escritura de argumentos (postura alternativa, conclusión y estructura), los que en conjunto explicaron el 57\% de la varianza. Es interesante observar que estos tres elementos que se agrupan en la tarea de producción representan la estructura dialéctica y dialógica que tiene un rol central en el modelo de calidad argumentativa que asume la 
tarea. En contraste, la justificación de un punto de vista (criterio 1), no formó parte de este componente; más aún, tuvo una carga mayor en el primer componente, aunque insuficiente $(<.4)$, y muy poca varianza compartida con los componentes, como se observa en los resultados de comunalidad. Cabe mencionar que, al probar una solución alternativa de tres componentes, en el tercero de estos solo cargó el criterio 1 , mostrando una alta saturación $(, 89)$.

Tabla 9.

Matriz ${ }^{\text {a }}$ de componente rotado para criterios evaluados

\begin{tabular}{|c|c|c|c|}
\hline \multirow[b]{2}{*}{ Criterios } & \multicolumn{3}{|l|}{ Componentes } \\
\hline & $\begin{array}{l}1 \\
\text { (Autovalor inicial }=2,76 \text {, } \\
34 \% \text { de varianza) }\end{array}$ & $\begin{array}{l}2 \\
\text { (Autovalor inicial = 1,81, } \\
23 \% \text { de varianza) }\end{array}$ & Comunalidad \\
\hline 1. Justificación & .30 & .17 &, 14 \\
\hline 2. Perspectiva alternativa &,- 08 & .67 &, 44 \\
\hline 3. Conclusión & .07 & 80 &, 66 \\
\hline 5. Estructura & .02 &, 94 &, 88 \\
\hline 6. Posición A & ,72 &,- 31 & .54 \\
\hline 7. Razones P A & ,71 & .15 & .57 \\
\hline 8. Posición B & ,82 & .02 & 69 \\
\hline 9. Razones P B & ,79 & .11 & .66 \\
\hline $\begin{array}{l}\text { Correlación } \\
\text { entre componentes }\end{array}$ &, 17 & & \\
\hline
\end{tabular}

Nota: Método de extracción: análisis de componentes principales y método de rotación Oblimin. Los valores en negrita corresponden a cargas mayores a ,4

De acuerdo a estos resultados, se continuó el análisis con los puntajes totales de la tarea de comprensión argumentativa (todos los criterios) y de producción dialógica-dialéctica de argumentos (perspectiva alternativa, conclusión y estructura), y se consideró por separado el puntaje en justificación de un punto de vista. Respecto a la consistencia interna de los componentes, la tarea de comprensión argumentativa mostró un alpha de Cronbach de $\alpha=, 77$, y el componente dialógico-dialéctico, un alpha de Cronbach de $\alpha=, 75$, lo que es considerado aceptable. Ahora bien, mientras los dos componentes se correlacionan baja pero significativamente entre sí $(r=.18$, $p=, 01)$, sorprendentemente, la justificación del punto de vista en tarea de escritura argumentativa no correlaciona significativamente con el componente dialógico-dialéctico de la escritura $(r=.12, p=, 07)$, pero sí con el componente de comprensión argumentativa $(r=.20, p=, 003)$. 
${ }_{¿}$ Existen diferencias significativas en estas sub-habilidades de acuerdo al género de los participantes?

En promedio, la muestra de estudiantes alcanzó un $41 \%$ en promedio de nivel de logro en formulación de posición y justificación de punto de vista $(M=1,64, D E$ $=0,67$, rango $=0-3$, rango teórico $=0-3)$; un $24 \%$ promedio de nivel logro en el componente dialéctico-dialógico de escritura argumentativa $(M=1,95, D E=1,74$, rango $=0-7$, rango teórico $=0-8)$; y un 49,4\% en el componente de comprensión de argumentos $(M=5,93, D E=3,12$, rango $=0-12$, rango teórico $=0$ - 12). A nivel descriptivo se observaron diferencias entre los promedios de hombres y mujeres en la justificación de un punto de vista $\left(M_{\text {mujeres }}=1,76, D E=0,63 ; M_{\text {hombres }}=1,50, D E\right.$ = 0,69), componente dialéctico-dialógico $\left(M_{\text {mujeres }}=2,29, D E=1,82 ; M_{\text {hombres }}=1,47\right.$, $D E=1,52)$ y comprensión de argumentos $\left(M_{\text {mujeres }}=6,28, D E=2,97 ; M_{\text {hombres }}=5,8, D S\right.$ $D E=3,26)$. Para analizar si estas diferencias por género observadas en el desempeño de las pruebas eran significativas, se llevaron a cabo modelos de regresión múltiple con método jerárquico controlando por el colegio de procedencia. Como muestra la tabla 10 , se observan diferencias significativas, aunque pequeñas, a favor de las mujeres en las variables de escritura, tanto en justificación del punto de vista $(\beta=$ $0,24, p=, 014,95 \%$ IC $[0,05,0,43])$, como en el componente dialéctico-dialógico $(\beta$ $=0,62, p=, 007,95 \%$ IC $[0,17,1,06])$, controlando por colegio de procedencia. En contraste, no se observaron diferencias significativas por género en el desempeño de la tarea de comprensión de argumentos, controlando por colegio.

Tabla 10.

Regresión lineal para las variables Justificación, Escritura dialéctica-dialógica y Comprensión argumentativa

\begin{tabular}{|c|c|c|c|c|c|c|c|c|c|}
\hline \multirow[b]{3}{*}{ Variable } & \multicolumn{6}{|c|}{ Tarea de Escritura } & \multirow{2}{*}{\multicolumn{3}{|c|}{$\begin{array}{l}\text { Tarea de Comprensión Argu- } \\
\text { mentativa }\end{array}$}} \\
\hline & \multicolumn{3}{|c|}{ Justificación } & \multicolumn{3}{|c|}{ Escritura dialéctica-dialógica } & & & \\
\hline & $R^{2}$ & $\Delta R^{2}$ & B & $\mathrm{R}^{2}$ & $\Delta \mathrm{R}^{2}$ & B & $\mathrm{R}^{2}$ & $\Delta R^{2}$ & B \\
\hline $\begin{array}{l}\text { Paso } 1 \\
\text { Colegio }\end{array}$ &,- 01 & & & 18 & & & .16 & & \\
\hline $\begin{array}{l}\text { Paso } 2 \\
\text { Colegio } \\
\text { Género (Mujer) }\end{array}$ & .01 & $.02^{*}$ & $0,24^{*}$ & 21 & $.03^{* *}$ & $0,62^{* *}$ & ,16 & .00 & 0,73 \\
\hline $\mathrm{N}$ & 228 & & & 228 & & & 215 & & \\
\hline
\end{tabular}

${ }^{*} p<, 05{ }^{* *} p<, 01{ }^{* * *} p<, 001$ 


\section{Discusión}

El objetivo del estudio fue describir las habilidades de comprensión y producción de argumentos escrita de estudiantes de sexto básico, y las relaciones entre estas sub-habilidades, adaptando dos tareas desarrolladas por Reznitskaya y Wilkinson (2019). En relación a la primera pregunta de investigación, los resultados son coherentes con lo reportado previamente por Larrain et al. (2014) y Larrain y Singer (2020) en estudiantes chilenos usando otro instrumento de evaluación: se observa una dificultad general de los y las estudiantes en la comprensión y producción argumentativa. Consistentemente, entonces, se observa que estudiantes de educación subvencionada de transición al segundo ciclo básico en su mayoría formulan una posición y una razón para apoyarla, pero un porcentaje importante lo hace con una premisa de baja calidad, mostrando importantes dificultades para articular distintas razones. Esto es coherente con la literatura disponible que muestra que estudiantes son progresivamente más capaces no solo de elaborar razones de mejor calidad sino de formular más de una razón en apoyo de un punto de vista (Stein y Bernas, 1999; Stein y Albro, 2001). También es coherente con la literatura disponible la dificultad expresada por estudiantes al formular y sostener perspectivas alternativas, lo que representa mayor dificultad que elaborar argumentos (Larrain et al., 2013; Glassner y Schwarz, 2005; Stein y Bernas, 1999), sugiriendo la participación de procesos psicológicos diferentes. Es decir, aun con diferentes formas de medición, los resultados son consistentes con el conocimiento disponible.

Respecto a la comprensión argumentativa, nuestros resultados también son coherentes con estudios nacionales que muestran las dificultades de estudiantes (Parodi, 2007): un porcentaje importante de estudiantes no identifican la posición de un texto y quienes lo hacen tienen dificultad para identificar más de tres razones para sostenerla. Esta dificultad sugiere limitaciones en la capacidad de retener y recordar más de tres razones en cada caso, que también han sido reportadas en estudiantes universitarios (Britt et al., 2008; Larson et al., 2004), especialmente cuando el objetivo es comprender, como es este caso (Diakidoy et al., 2017). Es interesante observar que la identificación de la postura alternativa y sus razones muestra un desempeño similar a lo anterior, lo que sugiere que la comprensión de argumentos y contra-argumentos no sería particularmente diferente y pondrían en procesos psicológicos equivalentes. Esto no ha sido reportado hasta ahora.

Respecto a la relación entre comprensión y producción de argumentos, nuestros resultados sugieren una relación compleja. Coherente con los resultados de Reznitskaya y Wilkinson (2019) y Parodi (2007) nuestros resultados muestran que aunque ambas sub-habilidades están relacionadas sistemáticamente, involucran pro- 
cesos y actividades diferentes. Es particularmente interesante notar que producir argumentos e identificar argumentos y contra-argumentos tienen mayor relación que producir argumentos y contra-argumentos. Esto se puede explicar probablemente por la dependencia que existe entre formular una posición fundada y comprender la controversia en la cual hay que tomar posición. Formular contra-argumentos, sin embargo, requiere representar e imaginar la oposición, lo que es particularmente distinto, y no solo requiere mayor capacidad cognitiva (memoria), sino capacidad metacognitiva (Muller Mirza, Perret-Clermont, Tartas y Iannaccone, 2009).

Respecto a las diferencias de género, es interesante observar que nuestros resultados son coherentes con los resultados de Larrain y Singer (2020) quienes reportan diferencias a favor de las mujeres en producción argumentativa, y son parcialmente coherentes con los resultados de Reznitskaya y Wilkinson (2019) quienes encuentran diferencias solo en una de sus muestras. Sin embargo, mientras Reznitskaya y Wilkinson (2019) encuentran diferencias tanto en la tarea de comprensión como de producción, nuestros resultados solo muestran diferencias en esta última. De esta manera, estas diferencias pueden estar circunscritas a la población estudiada. En cualquier caso, es una diferencia que merecen ser seriamente considerada para el diseño pedagógico, de manera de ofrecer oportunidades de desarrollo equitativas para hombres y mujeres.

Es importante observar que hay que ser cautos al interpretar estos resultados y extraer conclusiones respecto a la naturaleza de la relación entre las sub-habilidades, pues hay que considerar que el desempeño podría estar estrechamente relacionado con características contextuales de las tareas mismas, y el conocimiento, percepciones y valoraciones de los estudiantes respecto qué es una buena argumentación cuando se está elaborando una posición personal (Coirier et al., 1999; Leitao, 2003; Stein y Bernas, 1999). De acuerdo a Leitao (2003), el conocimiento de posiciones y razones contrarias respecto a un tema no impactaría directamente en la disposición de adolescentes a escribir ensayos que elaboren contra-argumentos, ya que podrían no concebirlos como recursos retóricos valiosos para sus propios propósitos sino que, al contrario, como recursos que afectan negativamente la fuerza y la aceptabilidad del propio punto de vista. De esta manera, en base a estos resultados, no podemos sostener, por ejemplo, que el bajo desempeño en formulación de contra-argumentos da cuenta de la falta de una habilidad, sino que es necesario atender a las tareas y a las condiciones en la que estas fueron tomadas. En este sentido, las instrucciones pueden haber afectado el bajo desempeño en el desarrollo de la perspectiva alternativa en la tarea de escritura. En esta se solicita explícitamente expresar la propia opinión dando razones y evidencias, y responder a personas que pueden tener puntos de vis- 
ta contrarios y, por último, se recuerda hacer una conclusión. La manera en que está formulada esta instrucción puede inducir, más que a integrar y desarrollar un punto de vista alternativo, a seguir entregando razones para apoyar la propia posición. Junto con esto, no se puede dar por descontado que el vocabulario de los estudiantes no afecta el desempeño, especialmente ligado a las palabras opinión, razones, conclusión, entre otras. Aunque las pruebas fueron piloteadas, hay una influencia que no puede ser descartada. Es importante, entonces, al leer los resultados y proyectar el uso de estas pruebas en el futuro, pensar en cómo se pueden fortalecer los aspectos de las tareas para andamiar de mejor manera la producción de contra-argumentos.

Ahora bien, los resultados son coherentes con lo que asumimos es la experiencia educativa de la mayoría de los y las estudiantes en Chile: más centrada en explicar, producir razones y justificar, que en criticar y contra-argumentar; y en comprender textos que en producirlos. Teniendo en consideración que la argumentación depende de la enseñanza y experiencias de participación en actividades argumentativas (Kuhn y Udell, 2003), nuestros resultados dibujan un panorama coherente con relevantes implicancias educativas. Dado que las habilidades argumentativas dependen altamente del contexto, para aumentar su transferencia se requiere una práctica intensiva (ver Crowell y Kuhn, 2014) y continuada que permita formar hábitos de pensamiento argumentativo. Esto demanda la coordinación de distintas disciplinas y niveles educativos, lo que a su vez requiere una firme voluntad social y política.

Por último, es preciso observar que el estudio contribuye al ámbito de la evaluación de argumentos, lo que es relevante dada la escasa existencia de discusión respecto a la evaluación de estas competencias. Aunque realizamos un proceso de traducción y adaptación, y como se ha discutido, los resultados sugieren que los instrumentos funcionarían en nuestra muestra con relevantes similitudes a las muestras reportadas por Reznitskaya y Wilkinson (2019). Esta consistencia permite pensar que estas pruebas pueden ser usadas con cierto grado de confiabilidad para informar sobre el desarrollo de habilidades argumentativas de adolescentes que cursan el segundo ciclo básico en Chile. Estas pruebas han seguido un largo y riguroso camino de desarrollo, que puede ser aprovechado en el contexto nacional y, quizá, de habla hispana. Esto es relevante, especialmente considerando la escasez de instrumentos confiables disponibles para medir comprensión y producción de argumentación escrita en escolares. 


\section{Trabajos citados}

Scardamalia, Marlene y Carl Bereiter. "Knowledge telling and knowledge transforming in written composition." Advances in applied psycholinguistics 2 (1987): 142-175.

Billig, Michael. Arguing and thinking: A rhetorical approach to social psychology. Cambridge University Press, 1996.

Britt, M. Anne, Christopher Kurby, Srikanth Dandotkar y Christopher Wolfe. "I agreed with what." Memory 45 (2008): 52-84

Chambliss, Marilyn J., y P. Karen Murphy. "Fourth and fifth graders representing the argument structure in written texts." Discourse Processes 34 (1) (2002): 91-115.

Coirier, Pierre, Jerry Andriessen y Lucile Chanquoy. "From Planning to Translating: The Specificity of Argumentative Writing". En Coirier, P. y Andriessen, J. Foundations of argumentative text processing. Amsterdam: Amsterdam University Press, 1999.

Crowell, Amanda y Deanna Kuhn. "Developing dialogic argumentation skills: A 3-year intervention study." Journal of Cognition and Development 15 (2) (2014): 363-381.

Diakidoy, Irene-Anna N., Melina C. Ioannou, y Stelios A. Christodoulou. "Reading argumentative texts: comprehension and evaluation goals and outcomes." Reading and Writing 30. (2017): 1869-1890

Felton, Mark K. "The development of discourse strategies in adolescent argumentation." Cognitive Development 19 (1) (2004): 35-52.

Felton, Mark, y Deanna Kuhn. "The development of argumentive discourse skill." Discourse processes 32.2-3 (2001): 135-153.

Field, Andy P., Jeremy Miles, y Zoë Field. Discovering statistics using R. London: Sage, 2012.

Frau-Meigs, Divina, Brian O’Neill, Alessandro Soriani y Vitor Tomá. Digital citizenship education: Volume 1: Overview and new perspectives. Council of Europe, 2017.

Gainer, Jesse. "Critical thinking: Foundational for digital literacies and democracy." Journal of Adolescent \& Adult Literacy 56 (1) (2012): 14-17.

Garcia-Mila, Merce, Sandra Gilabert, Sibekl Erduran y Mark Felton. "The effect of argumentative task goal on the quality of argumentative discourse." Science Education 97 (4) (2013): 497-523. 
$100-$ Cogency, Journal of reasoning and argumentation

Gilabert, Sandra, Merce Garcia-Mila y Mark K. Felton. "The effect of task instructions on students' use of repetition in argumentative discourse." International Journal of Science Education 35 (17) (2013): 2857-2878.

Golder, Caroline y Delphine Pouit, "For a debate to take place the topic must be debatable. Developmental evolution of the negotiation and debatability of arguments." En Coirier, P. y Andriessen, J. (eds) Foundations of argumentative text processing (pp. 137-148). Amsterdam: Amsterdam University Press, 1999.

Govier, Trudy. A practical study of argument. Canada: Wardsworth, 2010

Glassner, Amnon y Baruch B. Schwarz. "The antilogos ability to evaluate information supporting arguments." Learning and Instruction 15 (4) (2005): 353-375.

Hefter, Markus, Kirsten Berthold, Alexander Renkl, Werner Riess, Sebastian Smid y Stefan Fries. "Effects of a training intervention to foster argumentation skills while processing conflicting scientific positions." Instructional Science 42 (6) (2014): 929-947.

Iordanou, Kalypso, Deanna Kuhn, Flora Matos, Yuchen Shi y Laura Hemberger. "Learning by arguing." Learning and Instruction 63 (2019).

Huang, Francis L. "Alternatives to multilevel modeling for the analysis of clustered data." The Journal of Experimental Education 84.1 (2016): 175-196.

Knudson, Ruth E. "The development of written argumentation: An analysis and comparison of argumentative writing at four grade levels." Child study journal (1992).

Kuhn, Deanna. "Critical thinking as discourse." Human Development 62.3 (2019): 146-164.

Kuhn, Deanna, Nicole Zillmer, Amanda Crowell y Julia Zavala. "Developing norms of argumentation: Metacognitive, epistemological, and social dimensions of developing argumentive competence." Cognition and Instruction 31.4 (2013): 456-496.

Kuhn, Deanna y Amanda Crowell. "Dialogic argumentation as a vehicle for developing young adolescents' thinking." Psychological science 22 (4) (2011): 545-552.

Kuhn, Deanna, Wendy Goh, Kalypso Iordanou y David Schaefield. "Arguing on the computer: A microgenetic study of developing argument skills in a computer-supported environment." Child development 79.5 (2008): 1310-1328.

Kuhn, Deanna y Wadiya Udell. "The development of argument skills." Child Development 74 (5) (2003): 1245-1260.

Kuhn, Deanna, Victoria Shaw, y Mark Felton. "Effects of dyadic interaction on argumentive reasoning." Cognition and instruction 15 (3) (1997): 287-315. 
Kuhn, Deanna. The skills of argument. Cambridge University Press, 1991.

Larrain, A. y Vivian Singer. (2020). "Habilidades de argumentación de estudiantes de educación básica subvencionada en Chile y su relación con variables socio-educativas." Cogency 11.1-2.

Larrain, Antonia. "Group-work discussions and content knowledge gains: Argumentative inner speech as the missing link?." Learning, culture and social interaction 14 (2017): 67-78.

Larrain, Antonia, y Felipe Burrows. "Las pautas sí importan. Efecto del uso de pautas sobre la calidad de la escritura argumentativa en la universidad." Formación universitaria 13.1 (2020): 115-126.

Larrain, Antonia, Paulina Freire, Renato Moretti, Magdalena Requena y Belén Sabat. "¿ La Universidad de Chile promueve las habilidades de argumentación escrita: Un estudio exploratorio comparativo de estudiantes de educación universitaria y educación técnica." Calidad en la Educación 43 (2015): 201-228.

Larrain, Antonia, Paulina Freire, y Christine Howe. "Science teaching and argumentation: One-sided versus dialectical argumentation in Chilean middle-school science lessons." International Journal of Science Education 36.6 (2014): 1017-1036.

Larrain, Antonia, Paulina Freire y Trinidad Olivos. "Habilidades de argumentación escrita: Una propuesta de medición para estudiantes de quinto básico." Psicoperspectivas 13 (1) (2014): 94-107.

Larrain, Antonia, Paulina Freire y Victoria Lillo. "Contra-argumentación en la escuela primaria: lo que los estudiantes pueden aprender." Estudios de Psicología 34(2) (2013): 233-235.

Larson, Aaron A., M. Anne Britt y Christopher A. Kurby. "Improving students' evaluation of informal arguments." The Journal of Experimental Education 77.4 (2009): 339-366.

Larson, Meredith, M. Anne Britt, y Aaron A. Larson. "Disfluencies in comprehending argumentative texts." Reading Psychology 25 (3) (2004): 205-224.

Leitão, Selma. "Evaluating and selecting counterarguments: Studies of children's rhetorical awareness." Written Communication 20 (3) (2003): 269-306.

Leitão, Selma. "The potential of argument in knowledge building." Human development 43 (6) (2000): 332-360.

Marinkovich, Juana. "Las estrategias cognitivo-retóricas y la dimensión dialéctica de la argumentación oral en una clase de lengua castellana y comunicación." Revista signos 40 (63) (2007): 127-146. 
McNeill, Katherine L. y Joseph Krajcik. "Inquiry and scientific explanations: Helping students use evidence and reasoning." Science as inquiry in the secondary setting (2008): 121-134.

Mercer, Neil. "Developing argumentation: Lessons learned in the primary school." Argumentation and education. Springer, Boston, MA, 2009. 177-194.

Mercier, Hugo y Dan Sperber. "Why do humans reason? Arguments for an argumentative theory." (2011).

Muller Mirza, Annely Perret-Clermont, Valery Tartas y Antonio Iannaccone. Psychosocial processes in argumentation. En Muller Mirza, N. y Perret-Clermont, A. N. Argumentation and Education. New York: Springer, 2009.

Muñoz, Pablo, y Amaia Redondo. "Inequality and academic achievement in Chile." Cepal Review 109 (2013).

Newell, George E., Richard Beach, Jamie Smith y Jennifer VanDerHeide. "Teaching and learning argumentative reading and writing: A review of research." Reading Research Quarterly 46.3 (2011): 273-304.

Parodi, Giovanni. "Reading-writing connections: Discourse-oriented research." Reading and writing 20.3 (2007): 225-250.

Piolat, Annie, Jean-Yves Roussey y Anne Gombert. "The development of argumentative schema in writing." En Coirier, P. y Andriessen, J. (eds) Foundations of argumentative text processing (pp. 117-135). Amsterdam: Amsterdam University Press, 1999.

Preiss, David, Juan Carlos Castillo, Elena Grigorenko y Jorge Manzi. "Argumentative writing and academic achievement: A longitudinal study." Learning and Individual Differences 28 (2013): 204-211.

Preiss, David, Juan Carlos Castillo, Paulina Flotts y Ernesto San Martín. "Assessment of argumentative writing and critical thinking in higher education: Educational correlates and gender differences." Learning and Individual Differences 28 (2013): 193-203.

Preiss, David, Elisa Calcagni, Ana María Espinoza, Daniela Gómez, Valeska Grau, Valentina Guzmán, Magdalena Müller, Francisca Ramírez y Paulo Volante. "Buenas prácticas pedagógicas observadas en el aula de segundo ciclo básico en Chile." Psykhe 23 (2) (2014): 1-12.

Quintana, Rafael y Richard Correnti. "The right to argue: teaching and assessing everyday argumentation skills." Journal of Further and Higher Education 43.8 (2019): 1133-1151. 
Rapanta, Chrysi, Merce Garcia-Mila y Sandra Gilabert. "What is meant by argumentative competence? An integrative review of methods of analysis and assessment in education." Review of Educational Research 83 (4) (2013): 483-520.

Revelle, William. (2019). Psych: Procedures for Personality and Psychological Research [version 1.9.4]. Evanston, IL: Northwestern University, 2019.

Reznitskaya, Aline, Richard Anderson, Brian McNurlen, Kim Nguyen-Jahiel, Anthi Archodidou y So-Young Kim. "Influence of oral discussion on written argument." Discourse Processes 32.2-3 (2001): 155-175.

Reznitskaya, Alina y Ian Wilkinson. "Assessing production and comprehension of written arguments in the upper-elementary grades". Studia paedagogica 24.4 (2019): 63-84.

Reznitskaya, Alina y Maughn Gregory. "Student Thought and Classroom Language: Examining the Mechanisms of Change in Dialogic Teaching." Educational Psychologist 48 (2) (2013): 114-33.

Richter, Tobias y Johanna Maier. "Comprehension of multiple documents with conflicting information: A two-step model of validation." Educational Psychologist 52.3 (2017): 148-166.

Santibáñez, Cristian, y Dale Hample. "Orientations toward interpersonal arguing in Chile." Pragmatics. Quarterly Publication of the International Pragmatics Association (IPrA) 25(3) (2015): 453-476

Santos, Clara Maria y Selma Leitão Santos. "Good argument, content and contextual dimensions". En Coirier, P. y Andriessen, J. (eds) Foundations of argumentative text processing (pp. 75-96). Amsterdam: Amsterdam University Press, 1999.

Smith, Blaine E., Carita Kiili, y Merja Kauppinen. "Transmediating argumentation: Students composing across written essays and digital videos in higher education." Computers \& Education 102 (2016): 138-151.

Stein, Nancy L., y Elizabeth R. Albro. "The origins and nature of arguments: Studies in conflict understanding, emotion, and negotiation." Discourse processes 32.2-3 (2001): 113-133.

Stein, Nancy y Ronan Bernas. "The early emergence of argumentative knowledge and skill." En Coirier, P. y Andriessen, J. (eds) Foundations of argumentative text processing (pp. 97-116). Amsterdam: Amsterdam University Press, 1999.

Stein, Nancy y Christopher Miller. "A theory of argumentative understanding: Relationships among position preference, judgments of goodness, memory and reasoning." Argumentation 7 (2) (1993): 183-204. 
104 - Cogency, Journal of reasoning and argumentation

Schworm, Silke y Alexander Renkl. "Learning argumentation skills through the use of prompts for self-explaining examples." Journal of Educational Psychology 99 (2) (2007): 285.

Stephen, Toulmin. The uses of argument. Cambridge University Press, 1958.

van Eemeren, Franz y Rob Grootendorst. Argumentation, Communication and Fallacies. New Jersey: Lawrence Erlbaum Associates, 1992.

Vygotski, Lev S. "Pensamiento y Lenguaje". En Obras Escogidas II. Madrid: Visor, [1934]/2001.

Van den Broek, Paul. "Comprehension and memory of narrative texts: Inferences and coherence." En Gernsbacher, M.A. (1994). Handbook of psycholinguistics. Academic Press, 1994.

von der Mühlen, Sarah, Tobias Richter, Sebastian Schmid y Kirsten Berthold. "How to Improve Argumentation Comprehension in University Students: Experimental Test of a Training Approach." Instructional Science: An International Journal of the Learning Sciences 47 (2) (2019): 215-237.

Voss, James F., Fincher-Kiefer, Wiley y Silfies, "On the processing of arguments. " Argumentation 7 (2) (1993): 165-181.

Weinberger, Armin, Karsten Stegmann y Frank Fischer. "Learning to argue online: Scripted groups surpass individuals (unscripted groups do not)." Computers in Human Behavior 26 (4) (2010): 506-515.

Wolfe, Michael B., Shawna M. Tanner y Andrew R. Taylor. "Processing and representation of arguments in one-sided texts about disputed topics." Discourse Processes 50.7 (2013): 457-497. 